\title{
Pump-Controlled Actuators with Dump Valves
}

\author{
Samuel Kärnell ${ }^{1}$, Emil Fernlund ${ }^{2}$, Fabian Lagerstedt ${ }^{3}$, and Liselott Ericson ${ }^{1}$ \\ ${ }^{1}$ Division of Fluid and Mechatronic Systems, Linköping University, Linköping, Sweden \\ E-mail: samuel.karnell@liu.se, lislott.ericson@liu.se \\ ${ }^{2}$ Hudiksvalls Hydraulikkluster, Hudiksvall, Sweden \\ E-mail: emil.fernlund@huddig.se \\ ${ }^{3}$ Huddig, Hudiksvall, Sweden \\ E-mail: fabian.lagerstedt@ huddig.se
}

\begin{abstract}
The electrification trend means an increased focus on the energy efficiency of mobile hydraulic systems. In turn, this means an increased focus on so-called pumpcontrolled systems, where actuators are controlled by pump flow rather than valve throttling. However, one of the main problems with pump-controlled systems is matching the flow into the pump with its outlet flow. There are many ways of solving this problem, but the solutions tend to be rather bulky and not ideal from a service perspective, since they often rely on accumulators. This paper presents simple but novel pump-controlled concepts, without accumulators. The energy consumption of the presented concepts is analysed and compared to other concepts. The analysis is based on measurements taken on a backhoe loader and the results show that implementation of the presented concepts on the backhoe's boom actuator can reduce the total power consumption by 50-60\% compared to the original load sensing system. This can be compared with 55-65\%, which is the yield for an ideal, accumulatorbased, pump-controlled system on the boom.
\end{abstract}

Keywords: Pump-controlled systems, Backhoe loader, Electrification, Mobile Hydraulic systems

\section{Introduction}

Electrification of mobile machines is leading to a stronger focus on energy efficiency, not least because batteries tend to be heavy and expensive. To obtain high energy efficiency on a system level, it is important to minimise throttling losses due to differences in load pressures. This is usually an issue for linear actuators in particular, but there are several solutions, including use of hydraulic transformers [1] or multi-chamber cylinders [2]. Another alternative is to isolate consumers by using multiple pumps. The use of so-called pump-controlled actuators, where one pump is dedicated to a specific actuator, is therefore of great interest. Another appealing feature of pump-controlled systems is that they generally offer energy recuperation possibilities, which can reduce energy consumption significantly for applications where high loads are often lowered.

In other words, pump-controlled systems are promising, yet they are not commonly found on mobile machines.

\subsection{Contributions}

Many pump-controlled systems have been presented over the years. The focus has generally been on making the systems as efficient as possible and this has had a negative impact on the systems' complexity and maintenance requirements. In this paper, the trade-off between simplicity and energy efficiency is considered. Simple, lowmaintenance concepts for pump-controlled asymmetric actuators are presented and analysed. Results on energy consumption from a case study conducted on a backhoe loader are presented, with the presented concepts compared to other system concepts. 


\subsection{Pump-controlled systems}

The term pump-controlled system has already been mentioned plenty of times. It derives from the fact that the pump flow is used to control the motion of the actuator in such systems, unlike in conventional valve-controlled systems. The flow can be controlled by either the pump speed, the pump displacement or both. Previously, there was much focus on displacement-controlled concepts, but the electrification trend of the last years has shifted the focus towards speed control. However, both controls are principally very similar and a circuit for one control type can generally be applied to the other type as well. The main difference is that a boost pump, required for the displacement control, is often included in displacement-controlled circuits.

Pump-controlled systems have been in use for decades in the aircraft industry, [3]. The actuators used in that context are symmetrical. However, the compactness of asymmetrical cylinders generally makes them the preferred choice in mobile machines. The problem with pump-controlled asymmetrical cylinders is that the flow in to the cylinder is different from the flow that comes out. There are many solutions to this problem and an overview of the work that has been done can be found in [4]. That article presents a huge number of possible solutions to the differential flow problem, but also outlines common problems with mode switching oscillations and accurate flow matching, for example.

It is clear that the solution to the differential flow problem has not converged. However, a generalised schematic of one of the most studied pump-controlled architectures is illustrated in Fig. 1. The control valve block in the figure is often built up from passive components. It can represent an inverse shuttle valve or pilot operated check valves, for example, but there are also recent instances where active valves have been used [5]. Regardless of the control valve design, the basic idea is that an accumulator is used to take care of the differential flow. The accumulator either provides or receives flow to/from the pump or the cylinder, depending on the mode of operation. During an extension, the accumulator provides energy to the system, but during retraction, the energy flow moves in the other direction. If the components are assumed to be lossless and pressure drops over the control valves are neglected, this system is ideal from an energy point of view. It is therefore used as a reference system in this paper.

Unfortunately, there are drawbacks related to the circuit presented in Fig. 1. To minimise problems with cavitation and to increase the stiffness of the system, some kind of pressurised reservoir is required. Typically, an accumulator is used. Note that multiple actuators can share an accumulator [6]. However, this requires installation space as well as maintenance and it complicates servicing. Filtering and cooling can also be problematic. It is therefore relevant to consider other alternatives.

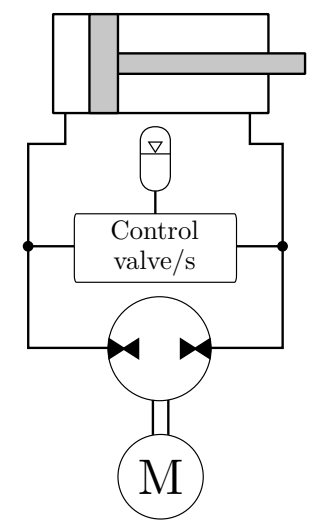

Figure 1: Common architecture for pump-controlled systems.

\section{The proposed concepts}

Instead of using an accumulator to handle the differential flow, the concepts that are presented in this paper share the feature that the required differential flow during extension comes from a pressure source and that the differential flow during retraction is directed to a tank through dump valves. The general architecture is illustrated in Fig 2. The dump valves are here built up from a pressure relief valve connected in series with an on/off-valve. The pressure relief valve takes care of the proportional control, and the on-board computer controls the on/off-valve. The opening pressure of the pressure relief valve is set slightly higher than the pressure source to avoid short circuit between the pressure source and dump valves. Regarding the pressure source, it can be either external or internal, which will be described more in section 2.1 and 2.2. The operation of the concepts in different modes is illustrated in Fig. 3. It can be seen how the pressure source supplies the low-pressure side of the system with flow during extension. During retraction, one of the dump valves is active instead. 


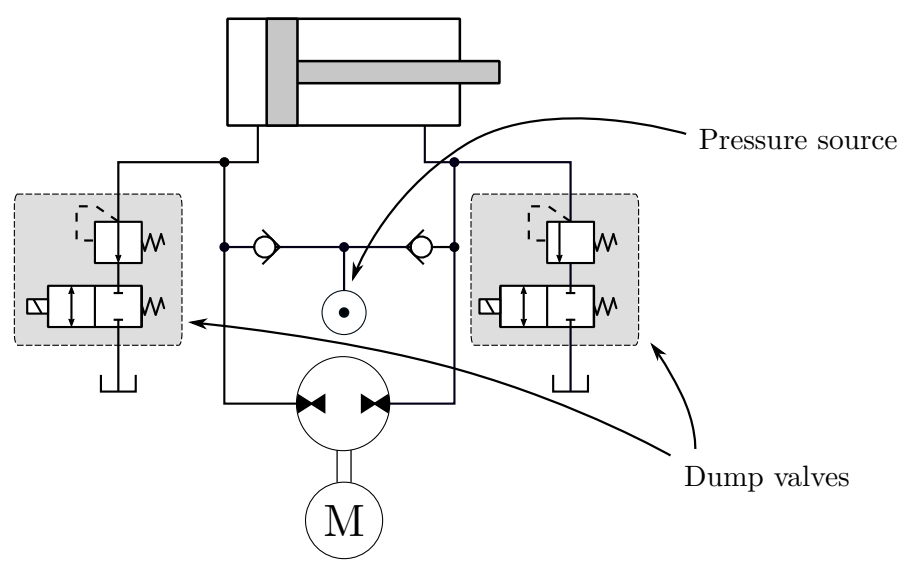

Figure 2: Generalised pump-controlled concept presented in this paper.

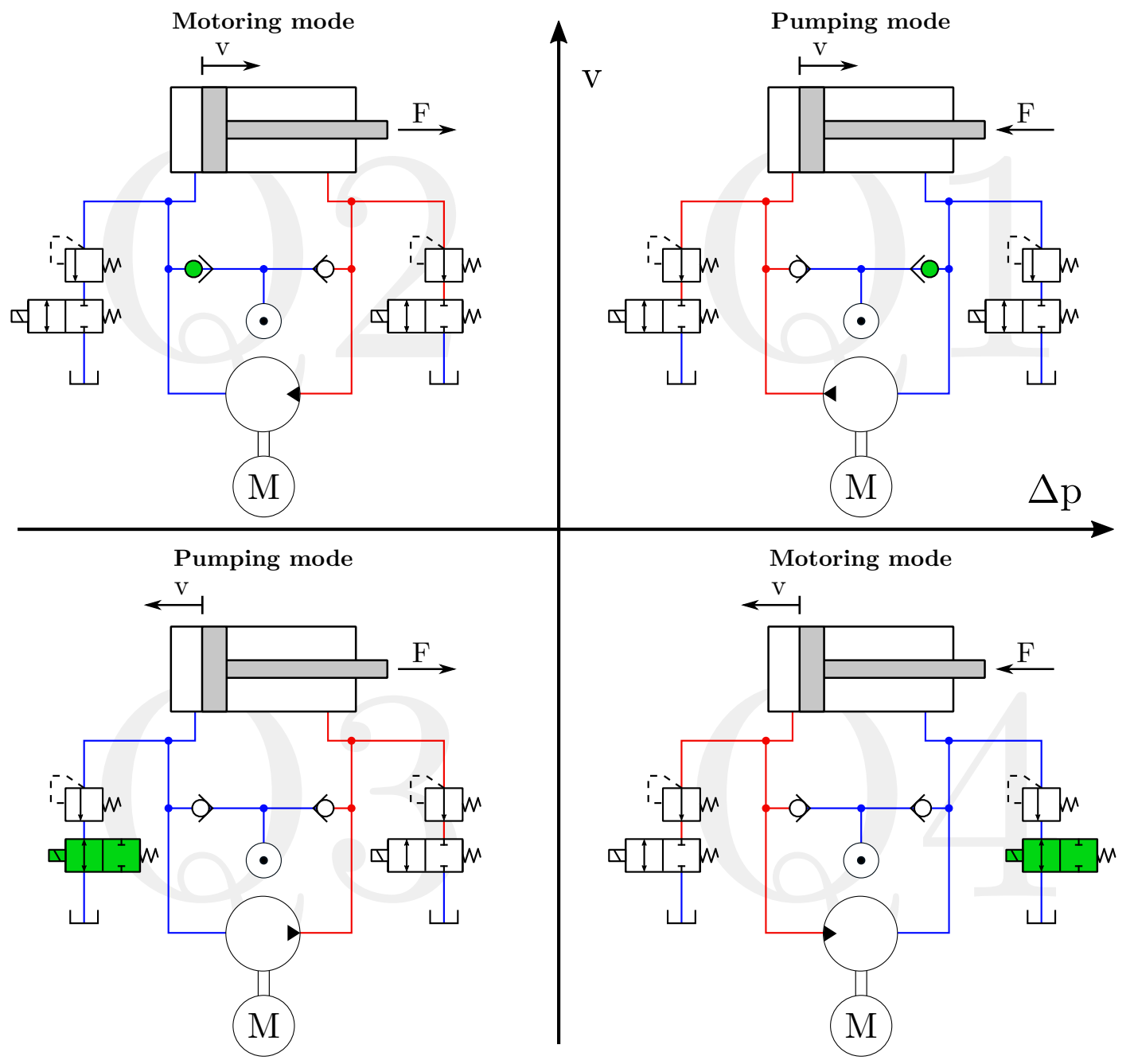

Figure 3: Working modes for the presented concept. The active control element for the differential flow is coloured. 


\subsection{Internal pressure supply}

The dumping process is equal to energy losses and the flow that is dumped during retraction must be refilled during extension. Figure 4 shows a system where internal pressure supply is used to supply flow during extension. This means that an internal charge pump is used to provide the required differential flow. Note that the required components for this concept are usually included in a traditional closed-circuit pump. However, it is important to remember that the charge pump is only allowed to pump fluid in one direction in this concept. This means that the direction of the cylinder must be controlled by means of the displacement or that the charge pump must be able to handle rotations in both directions without changing flow direction, which a wobble plate pump with check valves, for example, can do. Furthermore, for optimal efficiency, the ratio between the charge pump and the main pump must match the cylinder ratio, but also the flow required for displacement control might have to be considered. Also note that the ratio will vary a great deal if displacement control is used on the main pump, which is not the case when speed control is applied. In the analysis presented in section 3, the two controls are therefore separated.

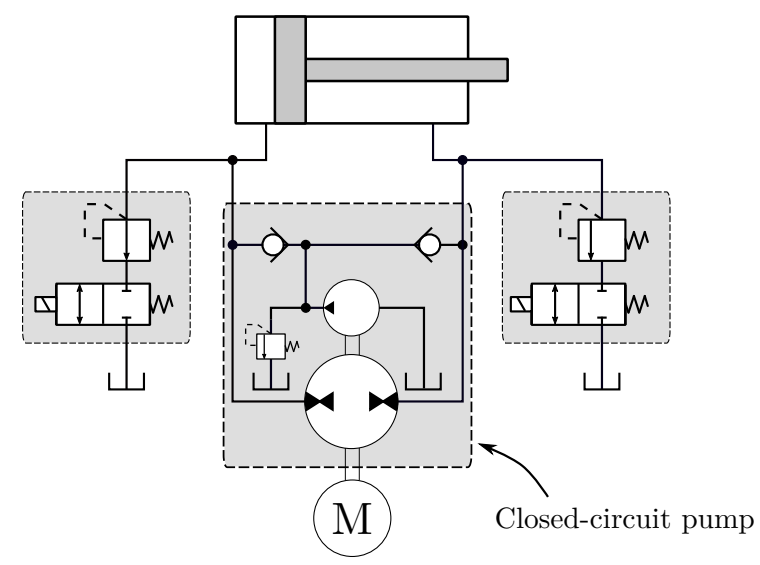

Figure 4: The presented concept with internal pressure supply.

This concept is similar in principle to displacement-controlled concepts that have been researched through the years (see e.g. [6-8]). The difference, apart from the lack of the accumulator, is that dump valves in combination with check valves are used instead of pilot operated check valves.

\subsection{External pressure supply}

For many mobile machines, it is only reasonable to implement pump-controlled actuators on a limited number of functions. This is mainly due to reasons of space and cost, for example, but it can also be due to limited energy saving possibilities for certain functions. Therefore, it is still relevant to power some functions with a more conventional valve-controlled system. This system can then be used as an external pressure supply for one or more pump-controlled systems. Pressure reducing valves can be used in-between the external pump and the pump-controlled systems to provide constant pressures to the latter. This is illustrated in Fig 5. The system within the block called Conventional system can be of any type, as long as it can provide the minimum pressure for the low-pressure side of the pump-controlled system. In this paper, a load sensing (LS) system is considered.

The usage of a pressure reducing valve can also be applied in systems that use pilot operated check valves or shuttle valves instead of check valves in combination with dump valves. This is because the pressure reducing valve can dump the excess flow to the tank. Efficiency-wise, the results are expected to be similar to the presented concept since they are only different ways of throttling the same flow. The difference is the pressure drop, which is set slightly higher in the dump valve. 


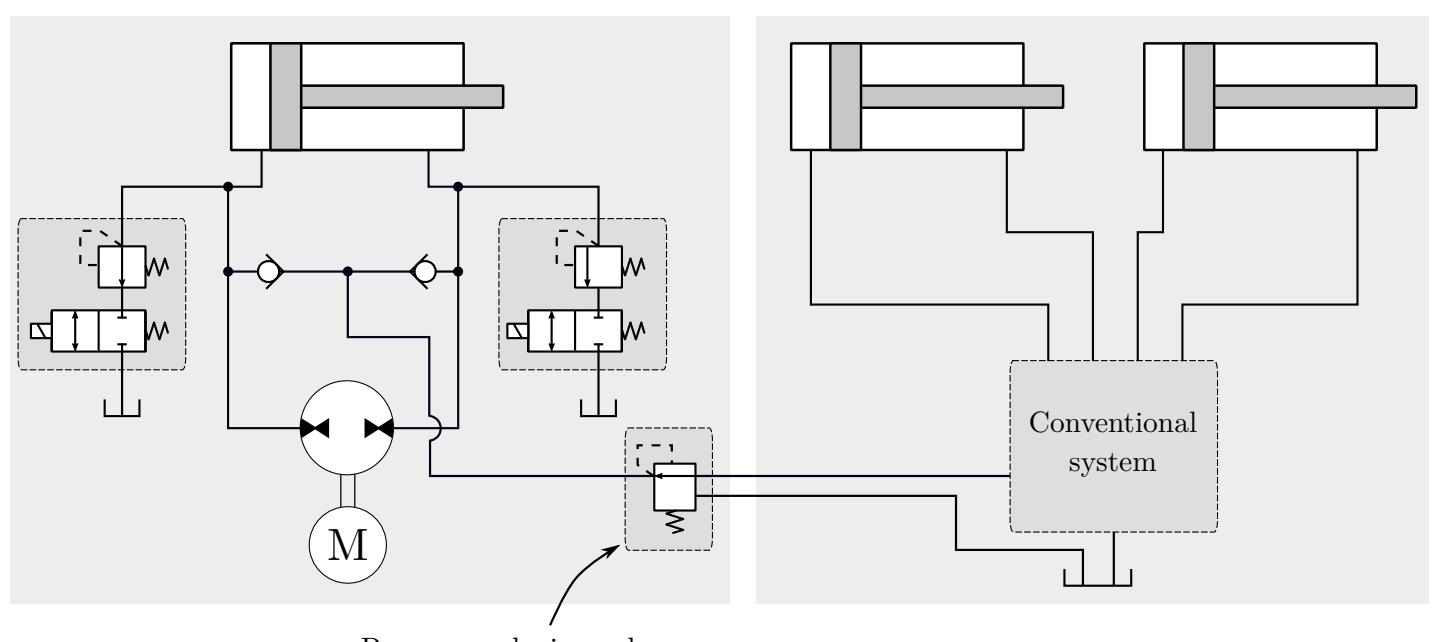

Pressure reducing valve

Figure 5: The presented concept with external pressure supply.

\section{Energy analysis}

This section contains an energy analysis, where the presented concepts are compared to other system architectures. The analysis is based on pressure and flow measurements taken on a backhoe loader in action for two different work tasks: excavating and grading. During the tasks, three of the backhoe's cylinders were used. An illustration of the backhoe loader and the cycles is presented in Fig. 6 and the measured pressure and flow requirements can be read from Fig. 7.

The energy consumption for the following systems is included in the analysis:

\section{- Conventional LS}

A conventional load sensing system is used for all three actuators. This is how the commercial backhoe loader is built today.

\section{- Separated LS}

The boom cylinder is powered by a separate LS system.

\section{- No recuperation}

The boom cylinder is pump-controlled, but no recuperation is possible. The arm and bucket are controlled by a conventional LS system. This system is included in the analysis to pinpoint the recuperation possibilities and does not represent any presented architecture.

\section{- Ideal pump-controlled system}

The boom cylinder is working in an ideal pump-controlled system according to system presented in Fig. 1. The arm and bucket are controlled by a conventional LS system.

\section{- Internal supply, speed-controlled}

The boom cylinder is working in a pump-controlled system with dump valves and internal pressure supply according to Fig. 4. The pump for the boom cylinder is speed-controlled, which means that the flow from the charge pump is proportional to the flow from the main pump. The arm and bucket are controlled by a conventional LS system.

\section{- Internal supply, displacement controlled}

The boom cylinder is working in a pump-controlled system with dump valves and internal pressure supply according to Fig. 4. The pump for the boom cylinder is displacement controlled, but the charge pump is fixed which means that the flow from the charge pump is constant and dimensioned for the maximum required differential flow. The arm and bucket are controlled by a conventional LS system.

\section{- External supply}

The boom cylinder is working in a pump-controlled system with dump valves and external pressure supply according to Fig. 5. The arm and bucket are controlled by a conventional LS system. 


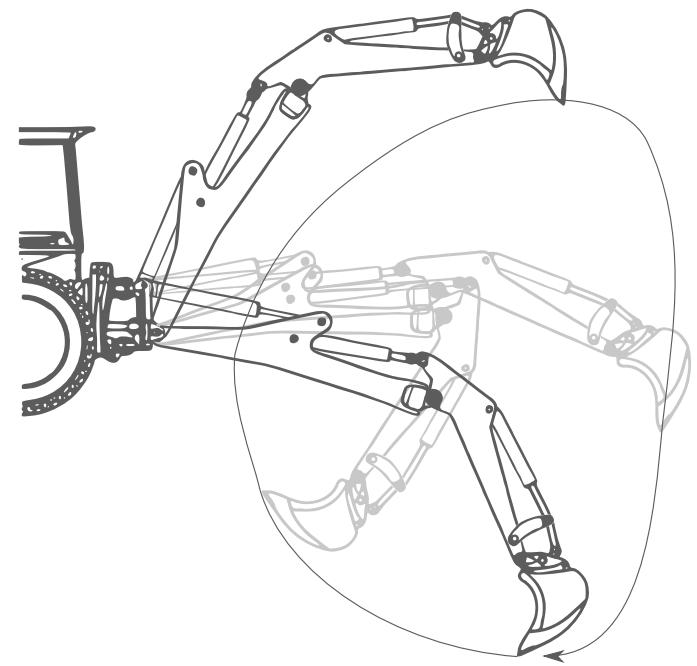

(a) Excavating cycle. Gravel was picked up from a low position and dropped from a high position.

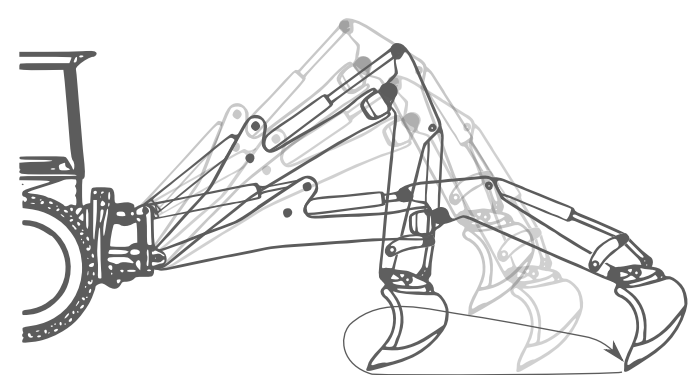

(b) Grading cycle with no additional load in the bucket.

Figure 6: Illustration of the example machine and its motion during the two analysed cycles. The cylinders for the boom, the arm and the bucket were operative during the cycles.
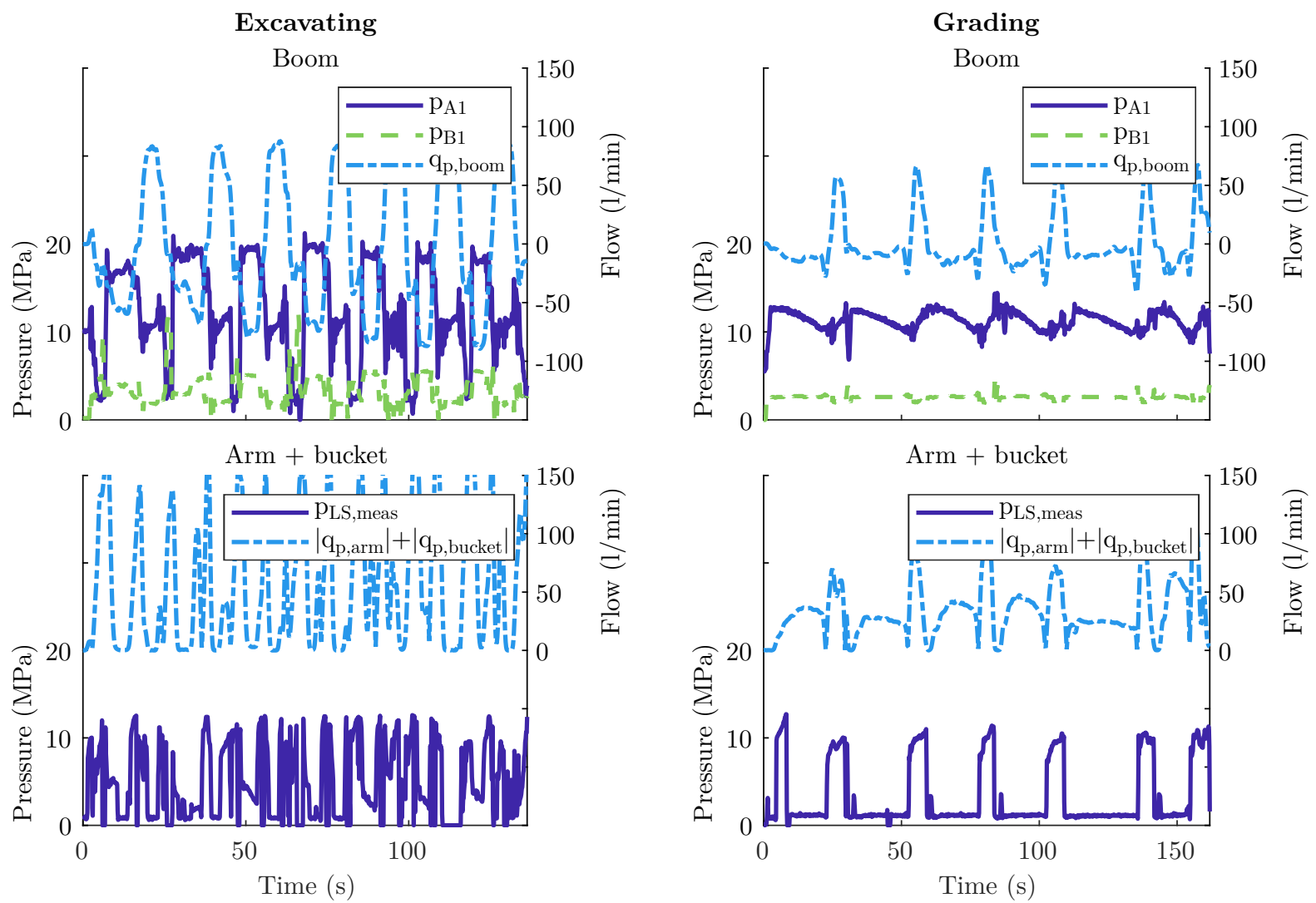

Figure 7: Measured pressure and flow for the two drive cycles. The left figures are for the excavating cycle and the right for the grading cycle. The labels correspond to the nomenclature in Fig. 8 and 9. 


\subsection{Measurement setup}

The measurements have been taken on a machine with the internal pressure supply concept implemented on the boom cylinder. The system architecture and positioning of sensors is illustrated in Fig. 8. For more information about the test machine, see [9].
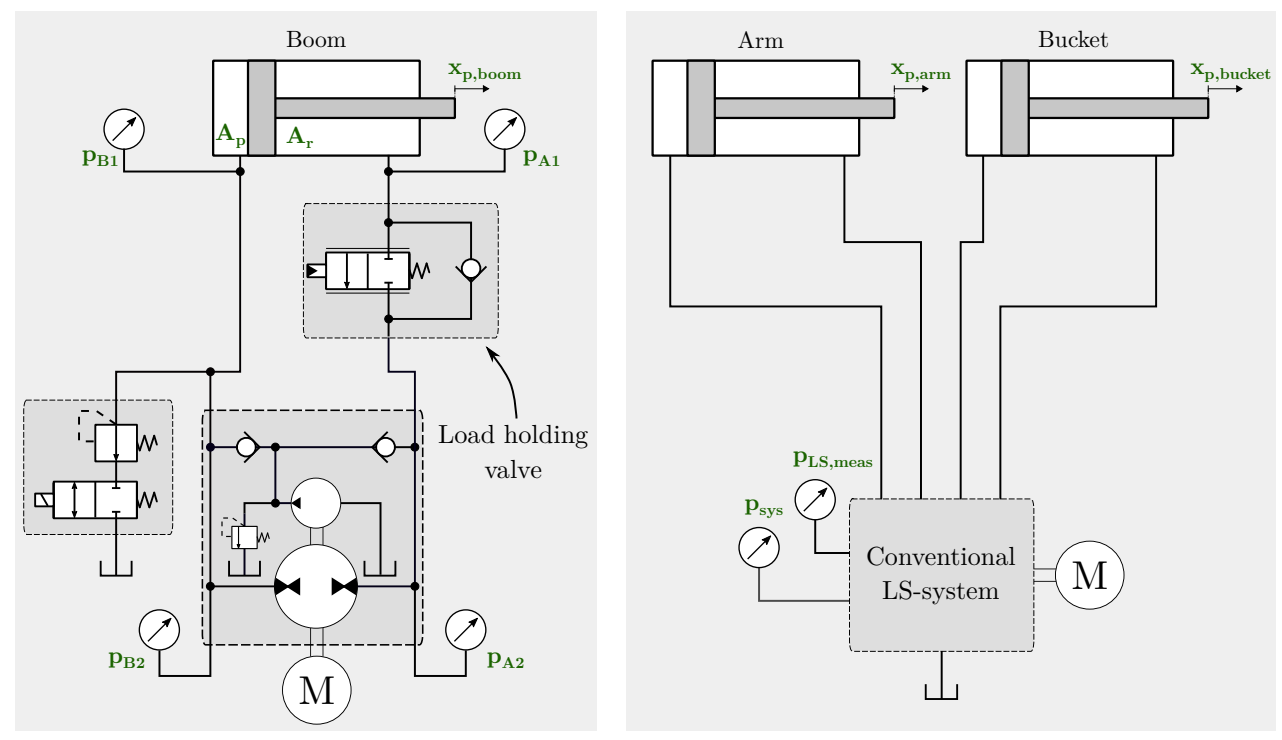

Figure 8: Measurement setup consisting of two separate circuits; one for the boom cylinder and one for the arm and bucket cylinders.

Note that the measurement setup only has one dump valve. This limits operation in quadrant 4 . However, that has not been considered a problem for the analysed test cases. Furthermore, the machine was equipped with a load holding valve, which is required for safety related reasons. This valve was controlled with pilot pressure and opened from joystick commands.

\subsection{Power calculations}

The approach for the power calculations is illustrated in Fig. 9.

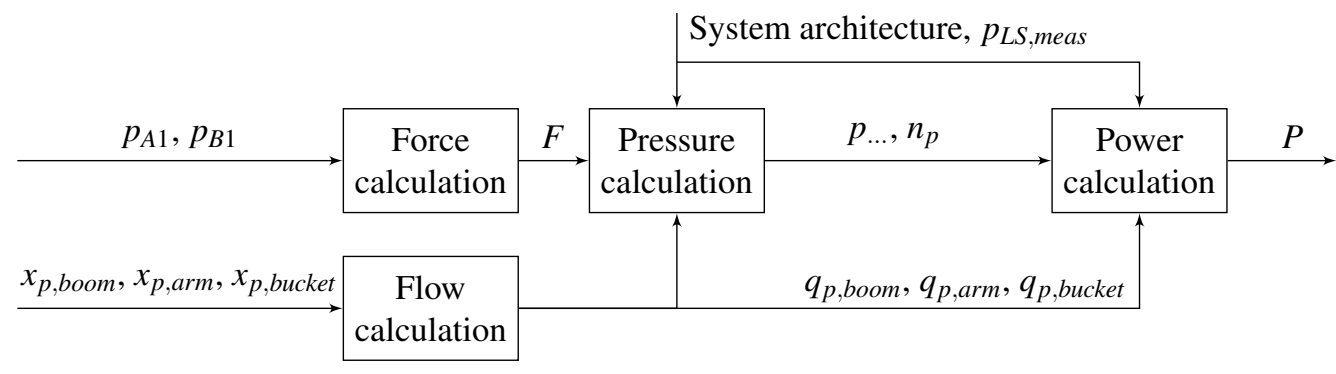

Figure 9: Power calculation process. The nomenclature relates to figure 8.

From the conducted measurements, an equivalent boom cylinder force was calculated according to Eq. (1).

$$
F=p_{B 1} A_{p}-p_{A 1} A_{r}
$$

This force was then used to recalculate pressures for the different system architectures. For the pressure calculations, the pressure on the low-pressure side was defined by the system concept itself, with consideration given to pressure drop over the load holding valve. The flow rates were calculated from the measured cylinder positions and the required pump speed was determined based on the flow rates. The power consumption for the different system concepts could then be calculated as follows. 


$$
\begin{aligned}
& P_{\text {conv. LS }}=\left(\max \left(p_{L S, \text { boom }}, p_{L S, \text { meas }}\right)+\Delta p_{L S}\right) \cdot\left|q_{p, \text { boom }}\right| \\
& +\left(\max \left(p_{L S, \text { boom }}, p_{L S, \text { meas }}\right)+\Delta p_{L S}\right) \cdot\left(\left|q_{p, \text { arm }}\right|+\left|q_{p, \text { bucket }}\right|\right) \\
& P_{\text {sep.LS }}=\left(p_{L S, \text { boom }}+\Delta p_{L S}\right) \cdot\left|q_{p, \text { boom }}\right| \\
& +\left(p_{L S, \text { meas }}+\Delta p_{L S}\right) \cdot\left(\left|q_{p, \text { arm }}\right|+\left|q_{p, \text { bucket }}\right|\right) \\
& P_{\text {noRecup }}=\max \left(\left(p_{B 2}-p_{A 2}\right) \cdot q_{p, \text { boom }}, 0\right) \\
& +\left(p_{L S, \text { meas }}+\Delta p_{L S}\right) \cdot\left(\left|q_{p, \text { arm }}\right|+\left|q_{p, \text { bucket }}\right|\right) \\
& P_{\text {ideal }}=\left(p_{B 2}-p_{A 2}\right) \cdot q_{p, \text { boom }} \\
& +\left(p_{L S, \text { meas }}+\Delta p_{L S}\right) \cdot\left(\left|q_{p, \text { arm }}\right|+\left|q_{p, \text { bucket }}\right|\right) \\
& P_{\text {intSup }}=\left(p_{B 2}-p_{A 2}\right) \cdot q_{p, \text { boom }} \\
& +\left(p_{L S, \text { meas }}+\Delta p_{L S}\right) \cdot\left(\left|q_{p, \text { arm }}\right|+\left|q_{p, \text { bucket }}\right|\right) \\
& +p_{\text {supply }} \cdot\left|n_{p} D_{c p}\right| \\
& P_{\text {extSup }}=\left(p_{B 2}-p_{A 2}\right) \cdot q_{p, \text { boom }} \\
& +\left(p_{L S, \text { meas }}+\Delta p_{L S}\right) \cdot\left(\left|q_{p, \text { arm }}\right|+\left|q_{p, \text { bucket }}\right|\right) \\
& +\max \left(\left(p_{L S, \text { meas }}+\Delta p_{L S}\right), p_{\text {supply }}\right) \cdot \max \left(q_{\text {diff }}, 0\right) \\
& +\max \left(p_{\text {supply }}-\left(p_{\text {LS,meas }}+\Delta p_{L S}\right), 0\right) \cdot\left(\left|q_{p, \text { arm }}\right|+\left|q_{p, \text { bucket }}\right|\right)
\end{aligned}
$$

The first row in (2a) to (2f) corresponds to the power related to the boom and the second to the power related to the arm and bucket. The additional rows, which exist in equations (2e) and (2f), corresponds to the power that relates to the pressure supply. A description of different parameters in the equations follows:

- $\boldsymbol{q}_{\boldsymbol{p}, \boldsymbol{x}}$ represents the flow that the machine/machines must handle to correspond to the speed and load of cylinder $x$.

- $p_{L S, \text { boom }}$ (not relevant for pump-controlled systems) is the calculated required LS pressure for the boom. When this pressure is calculated, it is assumed that the pressure in the low-pressure side of the cylinder is constant.

- $p_{L S, \text { meas }}$ is the measured LS pressure for the arm and bucket.

- $\Delta p_{L S}$ is the pump pressure margin for the LS pump/pumps.

- $\boldsymbol{p}_{\boldsymbol{B} 2}-\boldsymbol{p}_{\boldsymbol{A} \mathbf{2}}$ (only relevant for pump-controlled systems) is the pressure differential over the pump-controlled machine.

- $\boldsymbol{p}_{\text {supply }}$ is the pressure setting for the charging of the pump-controlled actuator.

- $\boldsymbol{n}_{\boldsymbol{p}} \boldsymbol{D}_{\boldsymbol{c} \boldsymbol{p}}$ corresponds to the flow from the charge pump in a system with internal pressure supply.

- $\boldsymbol{q}_{\text {diff }}=\left(A_{p}-A_{r}\right) \cdot v_{\text {boom }}$ is the differential flow for the boom cylinder. 


\section{Results}

Figure 10 shows the power consumption integrated over the cycles for the different system architectures. It also shows how the energy is distributed. The results are for $p_{\text {supply }}=2.5 \mathrm{MPa}$ and $\Delta p_{L S}=1.5 \mathrm{MPa}$. For the architectures with internal pressure supply, the charge pumps are optimally sized.
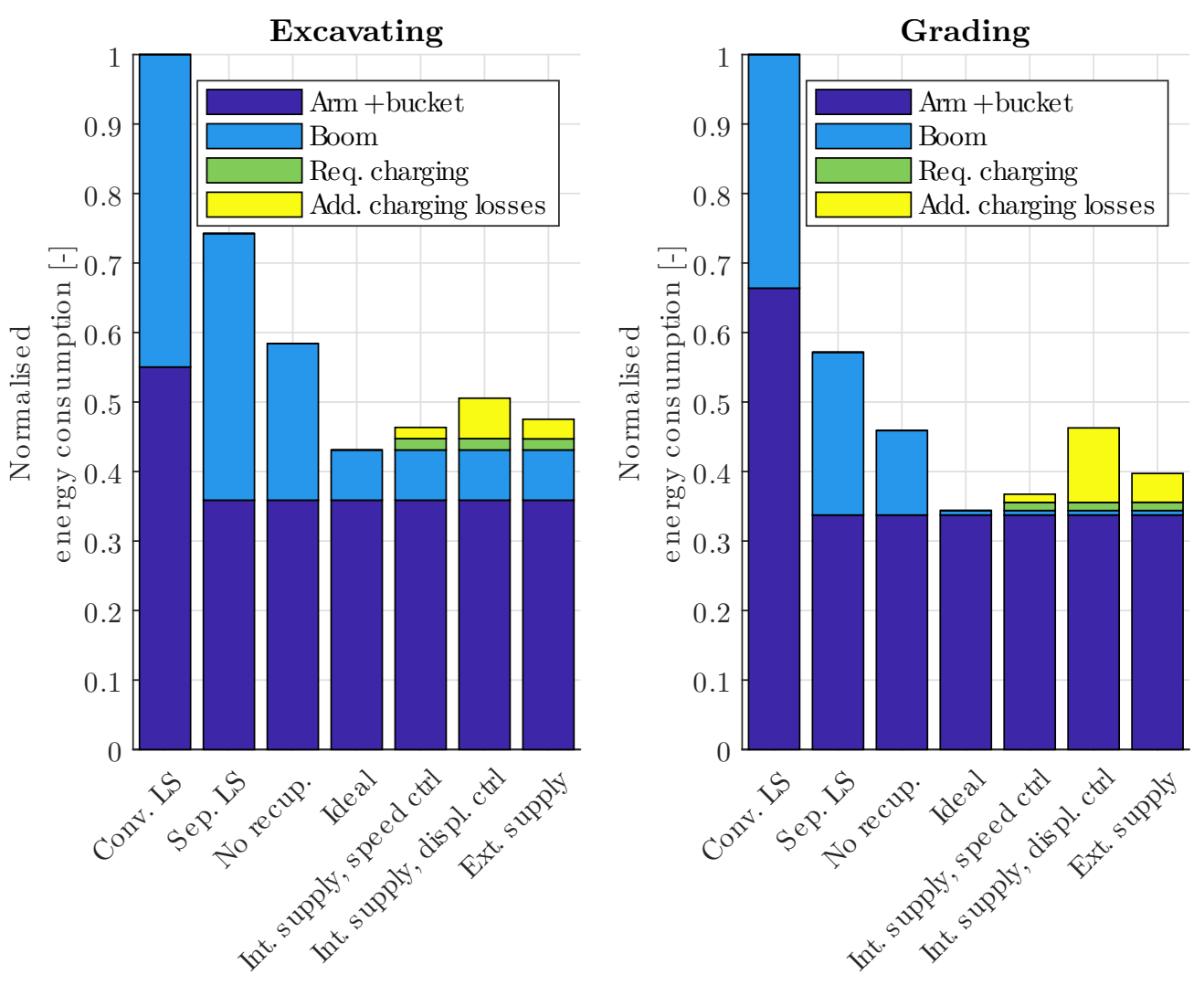

Figure 10: Normalised energy consumption for an excavating and a grading cycle for different system architectures.

The large differences between the conventional LS system and the separated LS system show that 25-40\% of the energy consumption is related to the mismatch in boom pressure and the pressure for other actuators. It can also be seen that the consumption for both the boom and arm + bucket decreases since the highest load alternates between the different functions during the cycle.

When comparing the separated LS system with the pump-controlled system with no recuperation, losses that relate to the pump pressure margin for the boom as well as losses related to the pressure in the low-pressure chamber can be seen. These losses correspond to about 10-15\% of the total consumption for the conventional LS system. Of this $10-15 \%$, the elimination of pump pressure margin losses corresponds to $30-40 \%$.

The difference between the no-recuperation system and the ideal pump-controlled system shows the recuperation possibilities. The results show that the relative recuperation possibilities are smaller for the excavating cycle than in the grading cycle. In the latter, the energy consumption for the boom is almost eliminated. That is, however, not the case for the excavating cycle since the load is dropped from a high vertical position. Still, it corresponds to more than $10 \%$ of the total energy consumption for the conventional system.

When it comes to the energy consumption for the internally and externally supplied systems, which are the concepts that this article is orbiting around, it can be seen that the charging-related losses can reach up to $10 \%$ of the consumption for the conventional system, but that the losses actually required only corresponds to $1-2 \%$. The required charging energy corresponds to the flow that is supplied to the system times the supply pressure. For concepts with internal pressure supply, the additional losses are related to the flow from the charge pump that is not delivered to the system. For external pressure supply, the additional losses are instead related to pressure drops. This means that concepts with internal pressure supply have losses that are proportional to the supply pressure setting (see Eq. (2e)), whilst the losses in the external pressure supply concept mainly depends on the drive cycle of the external pump. If it is working on pressure levels similar to the supply pressure setting, the losses are small. 


\section{Discussion}

The intention with this paper is to present concepts for pump-controlled systems based on dump valves. The dump valve concepts were developed to be simple, which means that they should be easy to implement and require low maintenance. It was obvious that the concepts would have worse efficiency than state of the art, accumulator-based, pump-controlled systems, but there is usually a trade-off between efficiency and simplicity. A quantification of the energy losses that comes with the concepts is therefore of interest. However, since mobile machines generally have widely varying drive cycles, this study shall only be considered as an example.

Regarding the simplicity, it is considered an advantage that no accumulator is needed in dump valve concepts. This is because accumulators are bulky, not free of maintenance and can complicate servicing in the field. Another advantage is that the concepts are not completely closed, which reduces problems related to e.g., air-bleeding, cooling, and filtering. The intention with the concepts was also that they were supposed to be built from common off-the-shelf components. That intention is, however, only partly fulfilled due to problems in finding optimal closed-circuit pumps for the concepts with internal pressure supply, which is described further below.

When it comes to energy efficiency, it could be seen that the displacement-controlled internal pressure supply system had the worst performance. The speed-controlled alternative was far better, but the problem with the speed-controlled system is that the charge pump must be able to handle speeds in both directions. The availability of pumps is therefore very limited, and the complexity of the system is increased. A solution could be to use combined speed- and displacement control, which would result in losses in-between pure speed- and displacement control. Nevertheless, it is important to mention that the charging- related energy consumption is proportional to the supply pressure in concepts with internal pressure control. In the example above, the supply pressure was set to $2.5 \mathrm{MPa}$, which is rather high. This could be reduced without any problem. A high supply pressure is, however, preferable from a controllability perspective since it means higher stiffness. Another way to reduce the losses is to address the losses that relates to the charge flow that is not supplied to the system. These could be reduced by adding a by-pass valve in the charging system that is activated in quadrant 3 and 4, but this solution would increase the complexity further and is therefore not considered as relevant.

The concept with external pressure supply is of considerable interest since it only requires a few, simple components, assuming that a conventional LS system is installed on the machine. No size matching between cylinder ratio and any charge pump needs to be considered, which makes the process of finding components simple compared to the internal pressure supply concepts. It will also have very good efficiency if the external pump mostly operates at pressures close to the supply pressure setting. That was, however, not the case in the analysed cycles.

When it comes to the design of the dump valves, it should be made clear that actively controlled proportional valves can be used instead of the solution with a pressure relief valve in series with an on/off-valve, which has been presented here. In fact, with an actively controlled proportional valve, the dump valve used in quadrant 4 can be omitted since high-pressure oil can be dumped on the piston side instead. Unfortunately, this would mean slightly worse efficiency, since less fluid will pass through the hydraulic machine, and also increased complexity.

Lastly, it should be stated that a demonstrator has been built as a proof of concept for a dump valve concept [9]. This machine used internal pressure supply. The drivability of the machine was considered to be satisfactory. Mode switching oscillations have not been analysed in detail, but it has not been considered an issue during the tests that have been performed. It should, however, be mentioned that switching between quadrant 1 and 2 is passive whilst switching between quadrant 3 and 4 is actively controlled. Oscillations in the latter case is therefore expected to be easier to avoid. Once again, it should also be stated that the presented solution with dump valves in combination with check valves can be replaced by pilot operated check valves, shuttle valves, or actively controlled valves. This would mean similar efficiency to the presented concepts, but with switching behaviour similar to earlier studied concepts $[8,10,11]$. Here, we do not make any statement on which is preferrable.

\section{Conclusions}

Pump-controlled systems based on dump valves are not optimal, but they can offer a good trade-off between efficiency and implementability. The concept with external pressure supply is considered highly interesting since it is easy to implement and since the efficiency is high if the external pressure supply usually operates at pressures similar to the supply pressure. Internal pressure supply concepts are less attractive since it can be hard to find a pump with the desired charge pump characteristics. 


\section{Nomenclature}

\begin{tabular}{lll}
\hline Designation & Denotation & Unit \\
\hline$D$ & Displacement & {$\left[\mathrm{m}^{3} / \mathrm{rev}\right]$} \\
$n$ & Rotational speed & {$[\mathrm{rpm}]$} \\
$P$ & Power & {$[\mathrm{W}]$} \\
$p$ & Pressure & {$[\mathrm{Pa}]$} \\
$q$ & Flow & {$\left[\mathrm{m}^{3} / \mathrm{s}\right]$} \\
\hline
\end{tabular}

\section{References}

[1] Vael Georges E. M., Achten Peter A. J., and Fu Zhao. The innas hydraulic transformer the key to the hydrostatic common pressure rail., 2000.

[2] Viktor Hristov Donkov, Torben Ole Andersen, Matti Linjama, and Morten Kjeld Ebbesen. Digital hydraulic technology for linear actuation: A state of the art review. International Journal of Fluid Power, 21(2), December 2020.

[3] Dominique Van Den Bossche. More electric control surface actuation; a standard for the next generation of transport aircraft. In Proceedings of the 10th European Conference on Power Electronics and Applications, pages $2-4,2003$.

[4] Søren Ketelsen, Damiano Padovani, Torben Ole Andersen, Morten Kjeld Ebbesen, and Lasse Schmidt. Classification and review of pump-controlled differential cylinder drives. Energies, 12(7):1293, 2019.

[5] Petter H. Gøytil, Damiano Padovani, and Michael R. Hansen. A novel solution for the elimination of mode switching in pump-controlled single-rod cylinders. Actuators, 9(1):20, Mar 2020.

[6] Monika Ivantysynova. Displacement controlled linear and rotary drives for mobile machines with automatic motion control. SAE transactions, pages 125-132, 2000.

[7] Christopher Williamson and Monika Ivantysynova. Pump mode prediction for four-quadrant velocity control of valueless hydraulic actuators. In Proceedings of the JFPS International Symposium on Fluid Power, volume 2008, pages 323-328. The Japan Fluid Power System Society, 2008.

[8] Longke Wang, Wayne J. Book, and James D. Huggins. A hydraulic circuit for single rod cylinders. Journal of Dynamic systems, Measurement, and Control, 134(1), 2012.

[9] Emil Fernlund. A novel pump-controlled asymmetric cylinder with electric regeneration: Implementation and evaluation of a closed hydraulic system on a backhoe. Master's thesis, 2020.

[10] C Williamson and M Ivantysynova. Stability and motion control of inertial loads with displacement controlled hydraulic actuators. In Proceedings of the 6th FPNI-PhD Symposium, West Lafayette, IN, USA, pages 15-19, 2010.

[11] Hakan Çalışkan, Tuna Balkan, and Bülent E Platin. A complete analysis and a novel solution for instability in pump controlled asymmetric actuators. Journal of Dynamic Systems, Measurement, and Control, 137(9), 2015. 